K. Tahara

Nagoya Math. J.

Vol. 69 (1978), 1-7

\title{
THE FOURTH DIMENSION SUBGROUPS AND POLYNOMIAL MAPS, II
}

\author{
KEN-ICHI TAHARA
}

\section{§1. Introduction}

In our previous paper [3] we proved the following ([3, Theorem 16]):

THEOREM A. Let $G$ be a 2-group of class 3. Let $G_{2}$ and $G / G_{2}$ be direct products of cyclic groups $\left\langle y_{q}\right\rangle$ of order $\alpha_{q}(1 \leqq q \leqq m)$, and of cyclic groups $\left\langle h_{i}\right\rangle$ of order $\beta_{i}(1 \leqq i \leqq n)$ with $\beta_{1} \geqq \beta_{2} \geqq \cdots \geqq \beta_{n}$, respectively. Let $x_{i}$ be representatives of $h_{i}(1 \leqq i \leqq n)$, and put $x_{i}^{\beta_{i}}=y_{1}^{c_{1}} y_{2}^{c_{13}}$ $\cdots y_{m}^{c_{m} m}(1 \leqq i \leqq n),\left[x_{j}, y_{s}\right]=y_{1}^{y_{1}^{j s}} y_{2}^{e^{j s}} \cdots y_{m}^{e_{m}^{j s}}(1 \leqq j \leqq n, 1 \leqq s \leqq m)$. Then $a$ homomorphism $\psi: G_{3} \rightarrow T$ can be extended to a polynomial map from $G$ to $T$ of degree $\leqq 4$ if and only if there exists an integral solution in the following linear equations of $X_{i q}(1 \leqq i \leqq n, 1 \leqq q \leqq m)$ with coefficients in $T$ :

$$
\begin{gathered}
\sum_{1 \leqq q \leqq m} e_{q q}^{j s} \frac{X_{i q}}{\left(\beta_{i}, \alpha_{q}\right)}=0 \quad(1 \leqq i, j \leqq n, 1 \leqq s \leqq m) \\
2^{\delta_{i j}}\left[\sum_{1 \leqq q \leqq m} c_{i q} \frac{X_{j q}}{\left(\beta_{j}, \alpha_{q}\right)}-\left(\frac{\beta_{i}}{\beta_{j}}\right)_{1 \leqq q \leqq m} \sum_{j q}\left\{\frac{X_{i q}}{\left(\beta_{i}, \alpha_{q}\right)}+\psi\left(\left[x_{i}, y_{q}\right]\right)\right\}\right]=0 \\
(1 \leqq i<j \leqq n),
\end{gathered}
$$

where $\delta_{i j}$ is the Kronecker symbol for $\beta_{i}$ : i.e. $\delta_{i j}=1$ or 0 according to $\beta_{i}=\beta_{j}$ or $\beta_{i}>\beta_{j}$, respectively.

As corollaries we had

COROLLARY 1 ([3, Corollaries 18 and 21$])$. If $2 \leqq n \leqq 3$ : i.e. the rank of $G / G_{2}$ is at most three, then $D_{4}(G)=G_{4}$.

In this paper we discuss the problem in the case $n \geqq 4$. We find out some sufficient conditions for $D_{4}(G)=G_{4}$ in the general case $n \geqq 4$, as the case such that the equations (I) and (II) in Theorem $\mathrm{A}$ have a

Received March 1, 1976. 
normal solution.*) We know only one counterexample to $D_{4}(G)=G_{4}$ due to Rips [2]. But we show that there exist infinitely many counterexamples to $D_{4}(G)=G_{4}$ in the case $n=4$, containing Rips' one as the simplest case.

\section{§2. General case $n \geqq 4$}

We determine some sufficient conditions for $D_{4}(G)=G_{4}$ in this general case $n \geqq 4$, as the case such that the equations (I) and (II) in Theorem A have a normal solution.

COROLlaRY 2. If $\left[x_{i}, x_{j}^{\beta_{i}}\right]^{2 \tilde{o}_{j} j}=1$ for $i<j$ with $1 \leqq i \leqq n-2$ : e.g. $\beta_{n-2} \geqq \alpha_{r}(1 \leqq r \leqq m)$, then $D_{4}(G)=G_{4}$.

Proof. Assume that $\left[x_{i}, x_{j}^{\beta_{i}}\right]^{2^{\delta i j}}=1$ and hence $2^{\delta_{i j}} \psi\left(\left[x_{i}, x_{j}^{\beta_{i}}\right]\right)=0$ $(i<j, 1 \leqq i \leqq n-2)$ for any homomorphism $\psi: G_{3} \rightarrow T$. Then it is easy to show by [3, Proposition 4] that $X_{i q}=0(1 \leqq i \leqq n-1,1 \leqq q \leqq m), X_{n q}$ $=-\left(\beta_{n}, \alpha_{q}\right) \psi\left(\left[x_{n}, y_{q}\right]\right)(1 \leqq q \leqq m)$ is an integral solution of the equations (I) and (II) in Theorem A, since $2^{\delta_{n-1, n}} \psi\left(\left[x_{n-1}, x_{n}^{\beta_{n-1}}\right]\right)=-2^{\delta_{n-1, n}} \psi\left(\left[x_{n}, x_{n-1}^{\beta_{n}-1}\right]\right)$. Now if $\beta_{n-2} \geqq \alpha_{r}(1 \leqq r \leqq m)$, then we have by [3, Proposition 4] for $i<j$ with $1 \leqq i \leqq n-2$,

$$
\begin{aligned}
2^{i i j} \psi\left(\left[x_{i}, x_{j}^{\beta_{i}}\right)\right] & =2^{\delta_{i j}}\left(\frac{\beta_{i}}{\beta_{j}}\right) \sum_{1 \leqq r \leqq m}\left(\sum_{1 \leqq q \leqq m} c_{j q} e_{r}^{i q}\right) \psi\left(y_{r}\right) \\
& =2^{\delta_{i j}}\left(\frac{\beta_{i}}{\beta_{j}}\right) \sum_{1 \leqq r \leqq m}\left\{\beta_{j} d_{r}^{i j}-\left(\frac{\beta_{j}}{2}\right) \sum_{1 \leqq q \leqq m} d_{q}^{i j} e_{r}^{j q}\right\} \psi\left(y_{r}\right) \\
& =2^{\delta_{i j}} \beta_{i} \sum_{1 \leqq q \leqq m} d_{r}^{i j} \psi\left(y_{r}\right) \\
& =0 .
\end{aligned}
$$

COROLlary 3. Assume that $\left[x_{i}, x_{j}^{\beta_{i}}\right]^{2 \tilde{o}_{i j}}=1$ for $i<j$ with $1 \leqq i \leqq n$ $-3:$ e.g. $\beta_{n-3} \geqq \alpha_{r}(1 \leqq r \leqq m)$. If any one of the following three conditions is satisfied, then $D_{4}(G)=G_{4}$ :

1) $\left[x_{n-2}, x_{n-1}^{\beta_{n}-2}\right]^{\delta_{n-2, n-1}}=1$

2) $\left[x_{n-2}, x_{n}^{\beta n-2}\right]^{2 \delta_{n-2, n}}=1$

3) $\left[x_{n-1}, x_{n}^{\beta_{n-1}}\right]^{2 \delta_{n-1, n}}=1$

Proof. Assume that $\left[x_{i}, x_{j}^{\beta_{i}}\right]^{\delta_{i j j}}=1$ and hence $2^{\delta_{i j}} \psi\left(\left[x_{i}, x_{j}^{\beta_{i}}\right]\right)=0$ $(i<j, 1 \leqq i \leqq n-3)$ for any homomorphism $\psi: G_{3} \rightarrow T$. Then it is easy to show by [3, Proposition 4] that $X_{i q}=0(1 \leqq i \leqq n-1,1 \leqq q \leqq m)$ and

\footnotetext{
*) See its definition in $[3, \S 6]$.
} 
$X_{n q}=-\left(\beta_{n}, \alpha_{q}\right) \psi\left(\left[x_{n}, y_{q}\right]\right)(1 \leqq q \leqq m)$ is an integral solution of (I) and (II) in the case 1$)$. In the case 2) $X_{i q}=0(1 \leqq i \leqq n-3,1 \leqq q \leqq m)$, $X_{n-2 q}=-\left(\beta_{n-2}, \alpha_{q}\right) \psi\left(\left[x_{n-2}, y_{q}\right]\right)(1 \leqq q \leqq m), X_{n-1 q}=0 \quad(1 \leqq q \leqq m)$ and $X_{n q}=-\left(\beta_{n}, \alpha_{q}\right) \psi\left(\left[x_{n}, y_{q}\right]\right)(1 \leqq q \leqq m)$ is their integral solution, and in the case 3) $X_{i q}=0(1 \leqq i \leqq n-3,1 \leqq q \leqq m), X_{n-2 q}=-\left(\beta_{n-2}, \alpha_{q}\right) \psi\left(\left[x_{n-2}\right.\right.$, $\left.\left.y_{q}\right]\right)(1 \leqq q \leqq m), X_{n-1 q}=0(1 \leqq q \leqq m), X_{n q}=-\left(\beta_{n}, \alpha_{q}\right) \psi\left(\left[x_{n}, y_{q}\right]\right)(1 \leqq q \leqq m)$ is their integral solution. Now if $\beta_{n-3} \geqq \alpha_{r}(1 \leqq r \leqq m)$, then we have by [3, Proposition 4] for $i<j$ with $1 \leqq i \leqq n-3$,

$$
\left[x_{i}, x_{j}^{\beta i}\right]^{2 \delta_{i j}}=1 \text {. }
$$

We may prove the following by a similar method of Corollary 6 below.

COROLlary 4. Assume that $\left[x_{i}, x_{j}^{\beta_{j}}\right]^{2 i_{i j}}=1$ for $i<j$ with $1 \leqq i \leqq n-4$ : e.g. $\beta_{n-4} \geqq \alpha_{r}(1 \leqq r \leqq m)$. If any one of the following seven conditions is satisfied, then $D_{4}(G)=G_{4}$.

1) $\left[x_{n-3}, x_{n-2}^{\beta_{n}-2}\right]^{\delta_{n-3, n-2}}=\left[x_{n-1}, x_{n}^{\beta_{n-1}}\right]^{\delta^{\delta_{n-1, n}}}=1$

2) $\left[x_{n-3}, x_{n-1}^{\beta_{n}-1}\right]^{\delta_{n-3, n-1}}=\left[x_{n-2}, x_{n}^{\beta_{n-2}}\right]^{2^{\delta_{n-2, n}}}=1$

3) $\left.\left[x_{n-3}, x_{n}^{\beta_{n-3}}\right]^{\delta^{\delta_{n-3, n}}}=\left[x_{n-2}, x_{n-1}^{\beta_{n}}\right]^{2}\right]^{\delta_{n-2, n-1}}=1$

4) $\left[x_{n-3}, x_{n-2}^{\beta_{n}-3}\right]^{\delta_{n-3, n-2}}=\left[x_{n-3}, x_{n-1}^{\beta_{n}-1}\right]^{\delta_{n-3, n-1}}=\left[x_{n-2}, x_{n-1}^{\beta_{n}-2}\right]^{\delta_{n-2, n-1}}=1$

5) $\left[x_{n-3}, x_{n-2}^{\beta_{n}-3}\right]^{2^{\delta_{n-3, n-2}}}=\left[x_{n-3}, x_{n}^{\beta_{n-3}}\right]^{2 \delta_{n-3, n}}=\left[x_{n-2}, x_{n}^{\beta_{n-2}}\right]^{2 \delta_{n-2, n}}=1$

6) $\left[x_{n-3}, x_{n-1}^{\beta_{n}-3}\right]^{2 \delta_{n-3, n-1}}=\left[x_{n-3}, x_{n}^{\beta_{n-3}}\right]^{2 \delta_{n-3, n}}=\left[x_{n-1}, x_{n}^{\beta_{n-1}}\right]^{2 \delta_{n-1, n}}=1$

7) $\left[x_{n-2}, x_{n-1}^{\beta_{n-1}^{2}}\right]^{2 \delta_{n-2, n-1}}=\left[x_{n-2}, x_{n}^{\beta_{n-2}}\right]^{2^{\delta_{n-2, n}}}=\left[x_{n-1}, x_{n}^{\beta_{n-1}}\right]^{2 \delta_{n-1, n}}=1$.

CoRollary 5. Let $n=2 \ell$ or $2 \ell+1$. If $\left[x_{i}, x_{j}^{\beta_{i}}\right]^{2 \delta_{i j}}=1$ for $1 \leqq i<j$ $\leqq \ell$ and $\ell+1 \leqq i<j \leqq n$, then $D_{4}(G)=G_{4}$.

Proof. Let $\psi: G_{3} \rightarrow T$ be any homomorphism. Then by [3, Proposition 4] we have that $X_{i q}=0(1 \leqq i \leqq \ell, 1 \leqq q \leqq m)$ and $X_{i q}=-\left(\beta_{i}, \alpha_{q}\right)$ $\psi\left(\left[x_{i}, y_{q}\right]\right)(\ell+1 \leqq i \leqq n, 1 \leqq q \leqq m)$ is an integral solution of (I) and (II) in Theorem A, since $2^{\delta_{i j}} \psi\left(\left[x_{i}, x_{j}^{\beta_{i}}\right]\right)=-2^{\delta_{i j}} \psi\left(\left[x_{j}, x_{i}^{\beta_{i}}\right]\right)$ for $\ell+1 \leqq i \leqq n$.

Q.E.D.

\section{§3. The case $n=4$}

In this case $n=4$ we show the following:

COROLLARY 6. If any one of the following seven conditions is satisfied, then $D_{4}(G)=G_{4}$; 
1) $\left[x_{1}, x_{2}^{\beta_{1}}\right]^{2_{12}}=\left[x_{3}, x_{4}^{\beta_{3}}\right]^{2 \delta_{s 4}}=1$

2) $\left[x_{1}, x_{3}^{\beta_{1}}\right]^{2 \delta_{13}}=\left[x_{2}, x_{4}^{\beta_{2}}\right]^{2 \delta_{24}}=1$

3) $\left[x_{1}, x_{4}^{\beta_{1}}\right]^{\delta_{14}}=\left[x_{2}, x_{3}^{\beta_{2}}\right]^{2 \delta_{23}}=1$

4) $\left[x_{1}, x_{2}^{\beta_{1}}\right]^{2^{\delta_{12}}}=\left[x_{1}, x_{3}^{\beta_{1}}\right]^{2_{13}}=\left[x_{2}, x_{3}^{\beta_{2}}\right]^{2 \delta_{23}}=1$

5) $\left[x_{1}, x_{2}^{\beta_{1}}\right]^{2 \delta_{12}}=\left[x_{1}, x_{4}^{\beta_{1}}\right]^{2^{\delta_{14}}}=\left[x_{2}, x_{4}^{\beta_{2}}\right]^{2^{\delta_{24}}}=1$

6) $\left[x_{1}, x_{3}^{\beta_{1}}\right]^{2 \delta_{13}}=\left[x_{1}, x_{4}^{\beta_{1}}\right]^{2^{\delta_{14}}}=\left[x_{3}, x_{4}^{\beta_{3}}\right]^{2 \delta_{34}}=1$

7) $\left[x_{2}, x_{3}^{\beta_{2}}\right]^{2 \delta_{23}}=\left[x_{2}, x_{4}^{\beta_{2}}\right]^{2 \delta_{24}}=\left[x_{3}, x_{4}^{\beta_{3}}\right]^{2 \delta_{34}}=1$.

Proof. Assume that $\left[x_{1}, x_{2}^{\beta_{1}}\right]^{2^{\delta_{12}}}=\left[x_{3}, x_{4}^{\beta_{8}}\right]^{2 \delta_{34}}=1$ and hence $2^{\delta_{12}} \psi\left(\left[x_{1}, x_{2}^{\beta_{1}}\right]\right)$ $=2^{\delta_{34}} \psi\left(\left[x_{3}, x_{4}^{\beta_{s}}\right]\right)=0$ for any homomorphism $\psi: G_{3} \rightarrow T$. Then $X_{i q}$ $=-\left(\beta_{i}, \alpha_{q}\right) \psi\left(\left[x_{i}, y_{q}\right]\right)(i=1,2 ; 1 \leqq q \leqq m), X_{i q}=0(i=3,4 ; 1 \leqq q \leqq m)$ is an integral solution of (I) and (II). In the remainder cases we list an integral solution corresponding in each case:

\begin{tabular}{c|cccc} 
Case & $X_{1 q}$ & $X_{2 q}$ & $X_{3 q}$ & $X_{4 q}$ \\
\hline 2$)$ & $*$ & 0 & $*$ & 0 \\
$3)$ & $*$ & 0 & 0 & $*$ \\
$4)$ & 0 & 0 & 0 & $*$ \\
$5)$ & 0 & 0 & $*$ & 0 \\
$6)$ & 0 & $*$ & 0 & 0 \\
$7)$ & $*$ & 0 & 0 & 0
\end{tabular}

where $*$ means $-\left(\beta_{i}, \alpha_{q}\right) \psi\left(\left[x_{i}, y_{q}\right]\right)$.

Q.E.D.

As a corollary of Corollary 6 we have

CoRollary 7. We have $D_{4}(G)=G_{4}$ in each case of the following three:

1) $\beta_{1} \geqq \beta_{2}=\beta_{3}=\beta_{4}$

2) $\beta_{1}=\beta_{2}>\beta_{3}=\beta_{4}$

3) $\beta_{1}=\beta_{2}=\beta_{3}>\beta_{4}$.

Proof. Its proof is very similar in each case. For example we prove it in the case 2$)$. We show that we may take $\psi\left(\left[x_{1}, x_{3}^{\beta_{1}}\right]\right)$ $=\psi\left(\left[x_{2}, x_{4}^{\beta_{2}}\right]\right)=0$ by a suitable base change of $\left\{h_{1}, h_{2}, h_{3}, h_{4}\right\}$. Let $\psi: G_{3} \rightarrow T$ be any homomorphism. For $1 \leqq i<j \leqq 4$ put $\psi\left(\left[x_{i}, x_{j}^{\beta_{i}}\right]\right)=A_{i j} / 2^{r_{i j}}$ with $A_{i f} \in Z$ and $\left(2, A_{i j}\right)=1$. Put $h_{1}^{*}=h_{1}, h_{2}^{*}=h_{1}^{a_{21}} h_{2}, h_{3}^{*}=h_{3}^{a_{33}} h_{4}^{a_{34}}$ and $h_{4}^{*}$ $=h_{3}^{a_{45}} h_{4}^{a_{44}}$ for an odd integer $a_{33} a_{44}-a_{34} a_{43}$, and put $x_{i}^{*}=\omega\left(h_{i}^{*}\right)(1 \leqq i \leqq 4)$. Then we have 


$$
\begin{aligned}
\psi\left(\left[x_{1}^{*}, x_{3}^{* \beta_{1}}\right]\right)= & a_{33} \psi\left(\left[x_{1}, x_{3}^{\beta_{1}}\right]\right)+a_{34} \psi\left(\left[x_{1}, x_{4}^{\beta_{1}}\right]\right) \\
\psi\left(\left[x_{2}^{*}, x_{4}^{* \beta_{2}}\right]\right)= & a_{21}\left\{a_{43} \psi\left(\left[x_{1}, x_{3}^{\beta_{1}}\right]\right)+a_{44} \psi\left(\left[x_{1}, x_{4}^{\beta_{1}}\right]\right)\right\} \\
& +a_{43} \psi\left(\left[x_{2}, x_{3}^{\beta_{2}}\right]\right)+a_{44} \psi\left(\left[x_{2}, x_{4}^{\beta_{2}}\right]\right)
\end{aligned}
$$

Therefore if $\gamma_{13}<\gamma_{14}$ and $\gamma_{23} \geqq \gamma_{24}$, or $\gamma_{13}=\gamma_{14}$ and $\gamma_{23} \neq \gamma_{24}$, or $\gamma_{13}>\gamma_{14}$ and $\gamma_{23} \leqq \gamma_{24}$, then we may choose $a_{21}, a_{33}, a_{34}, a_{43}$ and $a_{44}$ such that $\psi\left(\left[x_{1}^{*}, x_{3}^{* \beta 1}\right]\right)$ $=\psi\left(\left[x_{2}^{*}, x_{4}^{* \beta_{2}}\right]\right)=0, a_{21}=0$ and $a_{33} a_{44}-a_{34} a_{43}$ is odd. If $\gamma_{13}<\gamma_{14}$ and $\gamma_{14} \geqq \gamma_{24}$, or $\gamma_{13}=\gamma_{14}$ and $\gamma_{14} \leqq \gamma_{24}$, or $\gamma_{13}<\gamma_{14}$ and $\gamma_{13} \geqq \gamma_{23}$, then we may choose $a_{21}$, $a_{33}, a_{34}, a_{43}$ and $a_{44}$ such that $\psi\left(\left[x_{1}^{*}, x_{3}^{* \beta_{1}}\right]\right)=\psi\left(\left[x_{2}^{*}, x_{4}^{* \beta_{2}}\right]\right)=0$ and $a_{33} a_{44}$ $-a_{34} a_{43}$ is odd. Thus we may suppose that a) $\gamma_{13}<\gamma_{14}, \gamma_{23}<\gamma_{24}$ and $\gamma_{14}<\gamma_{24}$ : or b) $\gamma_{13}=\gamma_{14}, \gamma_{23}=\gamma_{24}$ and $\gamma_{14}<\gamma_{24}$ : or c) $\gamma_{13}<\gamma_{14}, \gamma_{23}>\gamma_{24}$ and $\gamma_{13}<\gamma_{23}$. In the case a) put $h_{1}^{*}=h_{1}^{a_{11}} h_{2}^{a_{12}}, h_{2}^{*}=h_{2}, h_{3}^{*}=h_{4}$ and $h_{4}^{*}=h_{3}^{a_{43}} h_{4}^{a_{44}}$ for odd integers $a_{11}$ and $a_{43}$. Then we have

$$
\begin{aligned}
& \psi\left(\left[x_{1}^{*}, x_{3}^{* \beta_{1}}\right]\right)=-a_{11} \psi\left(\left[x_{1}, x_{4}^{\beta_{1}}\right]\right)-a_{12} \psi\left(\left[x_{2}, x_{4}^{\beta_{2}}\right]\right) \\
& \psi\left(\left[x_{2}^{*}, x_{4}^{* \beta_{2}}\right]\right)=a_{43} \psi\left(\left[x_{2}, x_{3}^{\beta_{2}}\right]\right)+a_{44} \psi\left(\left[x_{2}, x_{4}^{\beta_{2}}\right]\right) .
\end{aligned}
$$

Therefore we may choose $a_{11}, a_{12}, a_{43}$ and $a_{44}$ such that $\psi\left(\left[x_{1}^{*}, x_{3}^{* \beta_{1}}\right]\right)$ $=\psi\left(\left[x_{2}^{*}, x_{4}^{* \beta_{2}}\right]\right)=0$ and $a_{11}, a_{43}$ are odd. In the case b) put $h_{1}^{*}=h_{2}, h_{2}^{*}$ $=h_{1}^{a_{21}} h_{2}^{a_{22}}, h_{3}^{*}=h_{3}^{a_{33}} h_{4}^{a_{34}}$ and $h_{4}^{*}=h_{4}$ for odd integers $a_{21}$ and $a_{33}$. Then we have

$$
\begin{aligned}
& \psi\left(\left[x_{1}^{*}, x_{3}^{* \beta_{1}}\right]\right)=-a_{33} \psi\left(\left[x_{2}, x_{3}^{\beta^{2}}\right]\right)-a_{34} \psi\left(\left[x_{2}, x_{4}^{\beta_{2}}\right]\right) \\
& \psi\left(\left[x_{2}^{*}, x_{4}^{* \beta_{2}}\right]\right)=a_{21} \psi\left(\left[x_{1}, x_{4}^{\beta_{1}}\right]\right)+a_{22} \psi\left(\left[x_{2}, x_{4}^{\beta_{2}}\right]\right),
\end{aligned}
$$

and hence we may choose $a_{21}, a_{22}, a_{33}$ and $a_{34}$ such that $\psi\left(\left[x_{1}^{*}, x_{3}^{* \beta_{1}}\right]\right)$ $=\psi\left(\left[x_{2}^{*}, x_{4}^{* \beta 2}\right]\right)=0, a_{21}$ and $a_{33}$ are odd. In the case c) put $h_{1}^{*}=h_{2}, h_{2}^{*}$ $=h_{1}^{a_{21}} h_{2}^{a_{22}}, h_{3}^{*}=h_{3}^{a_{33}} h_{4}^{a_{34}}$ and $h_{4}^{*}=h_{3}$ for odd integers $a_{21}$ and $a_{34}$. Then we have

$$
\begin{aligned}
& \psi\left(\left[x_{1}^{*}, x_{3}^{* \beta_{1}}\right]\right)=-a_{33} \psi\left(\left[x_{2}, x_{3}^{\beta_{2}}\right]\right)-a_{34} \psi\left(\left[x_{2}, x_{4}^{\beta_{2}}\right]\right) \\
& \psi\left(\left[x_{2}^{*}, x_{4}^{* \beta_{2}}\right]\right)=a_{21} \psi\left(\left[x_{1}, x_{3}^{\beta_{1}}\right]\right)+a_{22} \psi\left(\left[x_{2}, x_{3}^{\beta_{2}}\right]\right),
\end{aligned}
$$

and hence we may choose $a_{21}, a_{22}, a_{33}$ and $a_{34}$ such that $\psi\left(\left[x_{1}^{*}, x_{3}^{* \beta_{1}}\right]\right)$ $=\psi\left(\left[x_{2}^{*}, x_{4}^{* \beta_{2}}\right]\right)=0, a_{21}$ and $a_{34}$ are odd. Thus we may assume that $\psi\left(\left[x_{1}, x_{3}^{\beta_{1}}\right]\right)=\psi\left(\left[x_{2}, x_{4}^{\beta_{2}}\right]\right)=0$, and hence $D_{4}(G)=G_{4}$ Q.E.D.

Remark. Although in the case $\beta_{1}>\beta_{2}>\beta_{3}=\beta_{4}$, if $\beta_{1}=2 \beta_{2}$ or $\beta_{2}$ $=2 \beta_{3}$, then we may show that $D_{4}(G)=G_{4}$. Similarly in the case $\beta_{1}>\beta_{2}$ $=\beta_{3}>\beta_{4}$, if $\beta_{1}=2 \beta_{2}$ or $\beta_{3}=2 \beta_{4}$, then we may show that $D_{4}(G)=G_{4}$. Thus we conjecture that $D_{4}(G)=G_{4}$ in the both cases $\beta_{1}>\beta_{2}>\beta_{3}=\beta_{4}$ 
and $\beta_{1}>\beta_{2}=\beta_{3}>\beta_{4}$.

We construct infinitely many counterexamples to $D_{4}(G)=G_{4}$, whose order is $2^{8 k+22+\ell}$ with $k \geqq 2$ and $\ell \geqq 0$ in the case $\beta_{1} \geqq \beta_{2}>\beta_{3}>\beta_{4}$. In particular take $k=2$ and $\ell=0$, then this group is just the counterexample due to Rips [2].

Let $G$ be a 2-group of order $2^{8 k+22+\ell}$ satisfying the following:

1) $\alpha_{1}=2^{k+6}, \alpha_{2}=2^{k+4}, \alpha_{3}=2^{k+2}, \alpha_{4}=2^{k}$

2) $\beta_{1}=2^{k+4+\ell}, \beta_{2}=2^{k+4}, \beta_{3}=2^{k+2}, \beta_{4}=2^{k}$

3) $\left[x_{1}, x_{2}\right]=y_{1}^{2} y_{2},\left[x_{1}, x_{3}\right]=y_{1}^{-23} y_{3},\left[x_{1}, x_{4}\right]=y_{1}^{25} y_{4}$,

$\left[x_{2}, x_{3}\right]=y_{1},\left[x_{2}, x_{4}\right]=y_{1}^{2},\left[x_{3}, x_{4}\right]=y_{1}^{-2^{2}}$,

$\left[x_{1}, y_{q}\right]=1(1 \leqq q \leqq 4)$

$\left[x_{2}, y_{1}\right]=\left[x_{2}, y_{3}\right]=\left[x_{2}, y_{4}\right]=1,\left[x_{2}, y_{2}\right]=y_{1}^{22}$

$\left[x_{3}, y_{1}\right]=\left[x_{3}, y_{2}\right]=\left[x_{3}, y_{4}\right]=1,\left[x_{3}, y_{3}\right]=y_{1}^{-2^{4}}$

$\left[x_{4}, y_{1}\right]=\left[x_{4}, y_{2}\right]=\left[x_{4}, y_{3}\right]=1,\left[x_{4}, y_{4}\right]=y_{1}^{26}$

4) $x_{1}^{\beta_{1}}=y_{2}^{-2^{k+3+\ell}}, x_{2}^{\beta_{2}}=y_{3}^{2^{k}} y_{4}^{-2^{k-1}}, x_{3}^{\beta_{3}}=y_{2}^{2^{k}} y_{4}^{2^{k-2}}, x_{4}^{\beta_{4}}=y_{2}^{2^{k-1}} y_{3}^{2^{k-2}}$.

Then we may easily show that $G$ is a 2-group of class 3 . In this case the equations (I) and (II) in Theorem $\mathrm{A}$ are the following:

$$
\begin{aligned}
& 2^{2} \frac{X_{i 1}}{\beta_{i}}=0 \quad(1 \leqq i \leqq 4) \\
& 2^{\dot{o}_{12}}\left\{-\frac{X_{13}}{2^{2-\ell}}+\frac{X_{14}}{2^{1-\ell}}\right\}=0, \quad \frac{X_{12}}{2^{2-\ell}}=0 \\
& \frac{X_{33}}{4}-\frac{X_{34}}{2}-\frac{X_{22}}{4}-2^{k+4} \psi\left(y_{1}\right)=0 \\
& -\frac{X_{44}}{2}-\frac{X_{22}}{2}-2^{k+5} \psi\left(y_{1}\right)=0 \\
& \frac{X_{44}}{4}-\frac{X_{32}}{2}-\frac{X_{33}}{4}+2^{k+4} \psi\left(y_{1}\right)=0
\end{aligned}
$$

Taking $(1) \times 2+(2)+(3) \times 2$, we have

$$
2^{k+5} \psi\left(y_{1}\right)=\psi\left(y_{1}^{2 k+5}\right)=0
$$

and hence by [1, Proposition 4.1]

$$
D_{4}(G)=\left\{1, y_{1}^{2^{k+5}}\right\} \neq G_{4}=\{1\} .
$$

Thus we constructed a 2-group of order $2^{8 k+22+\ell}$ such that $D_{4}(G)=\left\{1, y_{1}^{2 k+5}\right\}$ $\neq\{1\}$ and $G_{4}=\{1\}$. 
In particular take $k=2$ and $\ell=0$, then this group is of order $2^{38}$, and we may show that this group is just equal to the counterexample due to Rips [2].

\section{REFERENCES}

[1] I. B. S. Passi, Dimension subgroups, J. Algebra 9 (1968), 152-182.

[2] E. Rips, On the fourth integer dimension subgroup, Israel J. Math. 12 (1972), $342-346$.

[ 3 ] K. Tahara, The fourth dimension subgroups and polynomial maps, J. Algebra 45 (1977), 102-131.

[4] - , On the structure of $Q_{3}(G)$ and the fourth dimension subgroups, Japan. J. Math. 3 (New Ser) (1977).

[5] —- Some remarks on the structure of $Q_{n}(G)$ for finitely generated groups G, to appear.

Aichi University of Education 